\title{
Le culte du cowboy et les figures du masculin à Kinshasa dans les années 1950
}

Manhood and The Cult of the Cowboy in 1950s Kinshasa

\section{Charles-Didier Gondola}

\section{(2) OpenEdition}

\section{Journals}

\section{Édition électronique}

URL : http://journals.openedition.org/etudesafricaines/17275

DOI : 10.4000/etudesafricaines. 17275

ISSN : $1777-5353$

Éditeur

Éditions de l'EHESS

\section{Édition imprimée}

Date de publication : 28 mai 2013

Pagination : 173-199

ISBN : 978-2-7132-2387-7

ISSN : 0008-0055

Référence électronique

Charles-Didier Gondola, "Le culte du cowboy et les figures du masculin à Kinshasa dans les années 1950 », Cahiers d'études africaines [En ligne], 209-210 | 2013, mis en ligne le 06 juin 2015, consulté le 16 juin 2020. URL : http://journals.openedition.org/etudesafricaines/17275 ; DOI : https://doi.org/ 10.4000/etudesafricaines. 17275

Ce document a été généré automatiquement le 16 juin 2020

(c) Cahiers d'Études africaines 


\title{
Le culte du cowboy et les figures du masculin à Kinshasa dans les années 1950
}

\author{
Manhood and The Cult of the Cowboy in 1950s Kinshasa
}

Charles-Didier Gondola

1 Dans son étude intitulée Poetics of Manhood, l'anthropologue américain Michael Herzfeld (1985 : 16) arpente le petit village crétois de Glendi et y décline l'impératif du masculin entre pratique et essence, différence qu'il clive dans les termes suivants: l'homme crétois se préoccupe davantage d'être bon à être un homme (being good at being a man) plutôt que d'être un homme bien (being a good man). La quête de la masculinité ne résiderait pas sui generis dans ce que les hommes font pour être des hommes (et pour paraître comme des hommes), mais plutôt dans la manière dont ils actent et performent la geste quotidienne du masculin à travers ce que Herzfeld appelle "performative excellence ». L'étude remarquable de Herzfeld est l'une des premières à reprendre pour le théoriser ce que l'on savait depuis fort longtemps, que les hommes jouent à être hommes; qu'il y a dans le masculin (comme dans le féminin d'ailleurs) non pas simplement une part de socialisation (d'où nous tenons la distinction faite par les premières études féministes entre sexe et genre), mais aussi une part de performance, de représentation et de théâtralisation. Pour d'autres chercheurs, il faut y ajouter une part de mystère. L'historien David Gilmore (1990:5), par exemple, assimile d'entrée de jeu le masculin (male gender identity) à un puzzle, une énigme, un cryptogramme insolvable. Le sentier qui y mène est semé d'embûches, d'essais manqués. Sans balises, le postulant s'oriente à l'aide de vagues admonitions et de normes culturelles, souvent éculées, s'évertuant à éviter les chausse-trappes qui menacent de le ramener à l'enfance (ibid. : 123).

2 Cette quête précaire du masculin, que Raphael Ray (1988) dit être une sorte de "makeshift masculinity» («masculinité de fortune»), se produit dans les enclaves homosociales, comme par exemple les cafés que Manuel Vale de Almeida (1996) décrit dans la petite ville portugaise de Pardais, les salles de cinéma coloniales de Dar es 
Salaam et de Kinshasa où s'élabore le culte du cowboy (Burton 2005 ; Gondola 2009), ou encore les rues dangereuses de Chicago (Diamond 2009), les usines et les cabarets des villes françaises du xIX ${ }^{e}$ siècle (Sohn 2009) ; tous constituent les fabriques par excellence des rituels du masculin. Autrement dit, le masculin ne s'oppose ni forcément ni intimement au féminin comme on a tendance à le penser, mais à des variantes " dégradées » et émasculées du masculin. Pierre Bourdieu (1998) articule cette idée dans son essai sur La domination masculine en montrant comment certaines formes de courage qu'exhibent les bandes de jeunes et que l'on retrouve au sein de quelques corps de métiers - il suffit de songer aux bâtisseurs des cathédrales ou des gratte-ciels newyorkais - où l'ouvrier brave le danger en faisant fi des mesures de prudence, "trouvent leur principe, paradoxalement, dans la peur» de "perdre la face». La crainte ici est de se voir ravaler par « les copains » dans la catégorie des « faibles", des " mauviettes", des « femmelettes », des « pédés ", etc. (ibid. : 73).

Qu'il existe donc une pluralité du masculin est un acquis fondamental de la recherche récente sur le masculin. Qu'il existe en revanche une hiérarchie des masculins reste une idée sans cesse passée au crible depuis l'ouvrage fondateur de la sociologue australienne R.W.Connell (1995) sur les masculinités. Prenant le contrepied des théories de genre qui jusqu'alors avaient tenu nature et culture en parfaite équidistance, Connell commence par réfuter la dimension biologique du masculin. Pour elle, le masculin résulte de configurations de pratiques générées dans des situations particulières et à l'intérieur d'un rhizome de relations sociales en mouvement perpétuel. Protéiforme et pluriel par définition, le masculin coïncide avec plusieurs autres catégories, notamment le statut social, le milieu d'origine et la classe d'âge. Partout, la variante du masculin qui domine est ce que Connell (ibid.: 79, 82) appelle "hegemonic masculinity", celle qui permet à certains hommes, dans certains milieux, d'accumuler pouvoir et privilèges et de recueillir des dividendes patriarcales.

Le concept de "hegemonic masculinity» a fait l'objet de plusieurs critiques (Cornwall \& Lindisfarne 1994; Howson 2006; Whitehead 2002) dont la mieux construite provient d'un élève même de Connell (Demetriou 2001). Empruntant à Homi Bhabha (1994: 37) sa dialectique gramcienne de "négociation au lieu de négation », Demetriou rejette la rigidité de l'approche de Connell et l'absence de "transactions». Au lieu d'une structure verticale cloisonnée dans laquelle "hegemonic masculinity» tiendrait le haut du pavé à des variantes subordonnées, Demetriou propose la notion de "bloc hybride " ("hybrid masculine bloc») qui combinerait éléments et pratiques de plusieurs masculinités. Un sujet donné (ou un groupe de sujets donnés) pourrait parfaitement combiner des éléments hétéroclites empruntés à plusieurs sources et traditions.

\section{Le Noir est-il homme?}

5 À Kinshasa, dans les années 1950, un certain nombre de jeunes Congolais ont justement combiné plusieurs éléments identitaires dans leur quête de paraître homme dans une société coloniale qui avait essentialisé, infantilisé, voire animalisé l'homme africain. « Hommes et frères? » s'interrogeait Baden Powell, le fondateur du mouvement scout, au sujet des Noirs. "Frères, peut-être, mais certainement pas hommes " (cité dans Uchendu 2008 : 1), prononçait-il avec la même certitude dogmatique d'un Hegel qui, au moment où les colonnes du Général Bugeaud s'apprêtaient à " pacifier » l'Algérie, avait réduit l'Africain à un «homme-animal » et son continent en un "pays de l'enfance». 
C'est à travers cette vision hégélienne de l'homme africain que les Belges se sont appliqué à soumettre le Congo à une politique paternaliste sans équivalent dans le reste du continent, hormis peut-être le régime d'apartheid en Afrique du Sud.

L'œuvre de civiliser les Noirs ayant été confiée aux missionnaires catholiques, à l'issue d'un concordat conclu entre Léopold II et le Vatican en 1906, ceux-ci se préoccupèrent exclusivement de l'homme noir, la femme noire ayant été disqualifiée comme musenzi ${ }^{1}$ (rustique). Un certain Robert Maistriaux (1957 : 191) l'accuse même d'être responsable du "capitis demunitio" des enfants noirs parce qu'elle a échoué dans son rôle de première éducatrice. Quant à l'homme noir, l'avis que distillent les doctes et que l'on retrouve simultanément dans la culture populaire et dans les conversations de table, le prononce "grand enfant». Alors que certains comme le psychologue français André Ombredane (1949) qui travaille au Congo Belge pour le compte de la CEMUBAC (Centre scientifique et médical de l'Université Libre de Bruxelles), attribuent l'« abrutissement » des Noirs à leur environnement oppressif, Maistriaux, lui, voit dans la «sous-évolution» des Noirs une corrélation entre psychologie et physiologie. Invoquant la pensée post-darwinienne telle qu'elle a été formulée dans les écrits de Lévy-Bruhl, Boas et Piaget, Maistriaux fait passer une batterie de tests cognitifs aux populations indigènes du Congo qu'il répartit en trois groupes distincts selon leur milieu de résidence : la population de la brousse (qu'il définit comme "sous-évoluée »), la population des centres-extra-coutumiers ${ }^{2}$, et les évolués ${ }^{3}$. Au sein des deux premières catégories, Maistriaux identifie une majorité de sujets dont les scores ne sont pas plus enviables que ceux des phrénasténiques européens.

7 Bien avant ces deux travaux, c'est la bande dessinée d'Hergé, Tintin au Congo, qui contribua plus que tout autre traité savant à vulgariser et à propager l'image du Noir infantile et «sous-évolué "4. Mais bien plus fascinant encore pour notre propos sur le masculin est cette juxtaposition dans l'album de Tintin entre d'un côté des Noirs nus et dévirilisés, bêtes et méchants, et, de l'autre, un Tintin intrépide, à la silhouette un tantinet androgyne, et dont la neutralité du genre (dans le sens de gender), au fil des pages, laisse songeur le lecteur avisé. Que faut-il également penser des missionnaires blancs, barbus et affublés de leur longue soutane blanche, inlassablement à pied d'œuvre, bâtisseurs de missions, d'écoles et d'hôpitaux, mais dont le sexe reste un mystère aux yeux des Africains ${ }^{5}$ ?

\section{Interventions missionnaires}

8 Ce sont justement à ces missionnaires vêtus de robes blanches que l'on doit l'introduction d'un élément iconique du masculin, le western américain, qu'une frange de la jeunesse « désœuvrée » de Kinshasa saisit immédiatement dans sa quête pour être homme.

9 À l'origine, les projections cinématographiques au Congo représentaient un antidote contre le mal du pays, les rigueurs et l'ennui de la vie européenne sous les tropiques. Dès 1911, un petit public avide d'Européens se retrouvait à des projections de films produits en France et aux États-Unis, films qui représentaient autant de fenêtres vers la civilisation (Ramirez \& Rolot 1985 : 20). Avec la fin de la Seconde Guerre mondiale, les missionnaires réalisent rapidement le potentiel éducatif du septième art. À cet effet, ils créent le Centre congolais d'action cinématographique catholique. Plusieurs missionnaires mettent en scène des acteurs africains et européens dans des films et des 
documentaires destinés à poursuivre sur le grand écran leur travail d'évangélisation et de civilisation. Le plus prolifique, l'abbé Cornil, arrivé au Congo en 1949 et qui soutient avoir appris le métier à Hollywood, produit près de quatre-vingts films destinés aux indigènes.

Jusqu'au début des années 1950, les missionnaires estiment encore indispensable d'exposer les indigènes, en milieu rural comme en ville, à des documentaires didactiques dont les titres - L'Heure (1947), L'Utilité de la couverture (1947), La Propreté du corps (1947), La Brouette (1948) et La Propreté de la maison (1950) - se passent de tout commentaire. Pour cette audience " primitive ", proclame un missionnaire, «il faut un cinéma à la mesure de son cerveau d'enfant » (cité dans Gondola 1999 : 177). Certaines projections donnent même lieu à des tohu-bohus, les spectateurs africains ne supportant plus la condescendance et le paternalisme des promoteurs européens ${ }^{6}$. À Ngiri Ngiri, l'un des quartiers de Kinshasa où comme nous le verrons le culte du cowboy bat son plein, des jeunes spectateurs protestent bruyamment à la sortie d'un documentaire intitulé La Chasse en AEF. L'administrateur local qui généralement surveille les projections surprend des jeunes regagnant leur quartier en pleine conversation : «Ezali malamu te!» («C'est nul !»), vitupèrent les jeunes cinéphiles. En 1957, dans un autre quartier de Kinshasa, à Kintambo, un autre repaire de bandes de cowboys, des jeunes spectateurs lancent des pierres en direction de l'écran lors d'une projection en plein air de l'abbé Cornil (Convents 2006: 100). Aux prises avec des spectateurs subversifs qui, à leur façon, détournent (dans le sens que lui donnent les situationnistes [Debord \& Wolman 1956]) ces projections cinématographiques de leurs intentions originelles, les missionnaires renoncent à reprogrammer Golgotha, le film de Julien Duvivier (1935) décrivant la passion du Christ. La scène du Christ émasculé, « dédéifié » et déshumanisé, croulant sous le poids de la croix et fouetté par un centurion romain, suscite des «makasi! makasi!» («[frappe] plus fort! plus fort!») qui fusent d'une audience déchaînée ${ }^{8}$.

\section{Buffalo Bill à Kinshasa}

11 Quand le western américain arrive à Kinshasa via la Belgique ${ }^{9}$, les missionnaires qui tiennent les rênes de la Commission de contrôle des films cinématographiques, autrement dit la censure, font d'abord montre d'une extrême vigilance et de beaucoup de suspicion. Après tout le western se prête mal à l'œuvre d'évangélisation et la figure $\mathrm{du}$ cowboy inspire peu confiance aux Pères blancs. C'était sans compter sur le personnage de Buffalo Bill qui quasiment à lui seul fait entrer le cowboy en odeur de sainteté.

Buffalo Bill, de son vrai nom William Frederick Cody (1846-1917), a incarné les valeurs d'une Amérique dont l'Ouest (et non l'Atlantique) a symbolisé pendant longtemps, suivant la thèse de l'historien Frederick Jackson Turner, une frontier, un espace mouvant, le point de rencontre entre civilisation et barbarie. Pour conquérir et domestiquer cette frontier mythique il a fallu des hommes aux nerfs d'acier, sans peur ni reproche, des hommes qui manient le pistolet avec autant d'adresse que de fréquence.

13 Parmi tous ces hommes se distingue la silhouette de Buffalo Bill qui finit par incarner à la fois le patriotisme d'une Amérique guidée par son manifest destiny - l'idée d'un peuple indomptable et exceptionnel, voué à s'étendre jusqu'au Pacifique - et toutes les 
valeurs mâles associées à la vie dans l'Ouest-américain. Le président Theodore Roosevelt, qui accepta la vice-présidence honoraire du Buffalo Bill Memorial Association, l'immortalise dans un dithyrambe comme «le plus illustre de ces hommes à la trempe de fer et au caractère d'acier, dont le courage a ouvert l'ouest à l'établissement des colons et à la civilisation [...]. Plus Américain qu'Américain [...] il incarna ces traits de courage, de force et d'audace qui sont vitaux au bien-être de la nation » (cité dans Carter $2000: 2$ ). Considéré même par certains de ses contemporains comme un "messie païen" (Calder 1974: 191), Buffalo Bill, parmi ses nombreux exploits, a transformé le cowboy, décrié avant la Guerre civile et traité de "saddle tramp " (" clochard en selle»), en un centaure à la gâchette rapide. Il l'a accompli non seulement en sillonnant les grandes plaines de l'Ouest, mais également en se représentant sur scène où il fut l'un des seuls héros du Far West à jouer son propre personnage. Après un succès époustouflant en Amérique, il amène son Wild West Show en Europe et se produit devant le président français Sadi Carnot, le Pape Léon XIII et la Reine Victoria. Il se plaça ainsi, d'une part, dans l'Ouest au moment où la frontier s'amenuisait comme une peau de chagrin pour finalement disparaitre et, d'autre part, dans la représentation de l'Ouest à la tête d'une troupe bigarrée composée de cowboys, de Sioux (y compris le chef vaincu Sitting Bull) et d'un assortiment hétéroclite d'acrobates, de tireurs d'élite et de lanceurs de couteaux ${ }^{10}$.

Le personnage incarné par Cody rencontre un écho favorable chez les missionnaires belges qui n'ont peine à voir se profiler derrière la silhouette équestre de Buffalo Bill la figure prométhéenne du "Koning-Bouwer » (« le roi bâtisseur »), Léopold II, le fondateur du Congo. Outre les similarités physiques (les deux portent une barbe blanche drue de patriarche et sont souvent représentés chevauchant augustement leur monture), Buffalo Bill et Léopold II participent, chacun de leur côté, à faire reculer les frontières de la barbarie ${ }^{11}$. Presque simultanément, l'un répand la Civilisation dans les grandes plaines arides de l'Ouest à coup de revolver tandis que l'autre la fait pénétrer au « cœur des ténèbres » à coup de machette.

15 À Kinshasa, la popularité de Buffalo Bill est attestée à travers plusieurs «nuits du Far West ", organisées par les Européens dans les cercles hippiques de la ville dans les années 1950, et reconstituant le Wild West Show dans ses moindres détails : cowboys, chevaux, rodéo, concours de tir, saloon, feux de camps et, pour agrémenter la fête, " cowgirls» (Convents $2006: 170$ ).

16 Buffalo Bill fait irruption dans les quartiers africains à travers le film de William A. Wellman, Buffalo Bill, sorti en salle aux États-Unis en 1944 et en France et en Belgique en 1947. D'autres représentations cinématographiques de Buffalo Bill, notamment le film de Jerry Hopper, Le Triomphe de Buffalo Bill ${ }^{12}$ (Pony Express, 1953), dans lequel l'acteur Charlton Heston incarne le rôle de Buffalo Bill, fournit à cette jeunesse marginalisée une grammaire gestuelle et toute une sémiotique du masculin. D'autres films comme Les Desperados (The Desperadoes, 1943), La Vallée maudite (Gunfighters, 1947), Retour des sans loi (Return of the Bad Men, 1948), La Charge héroïque (She Wore a Yellow Ribbon, 1949) et un grand nombre de films westerns de série в leur apprennent la démarche du « dur-à-cuir ». Cette liste serait incomplète si n'y figurait la série télévisée américaine The Lone Ranger (doublée en français sous le titre Le Dernier des fédérés).

17 Il y aurait beaucoup à dire sur la façon dont ces jeunes regardent le western. Dans un essai sur l'oralité et le film et à l'aide d'exemples tirés du Congo Belge, Vincent Bouchard s'appesantit sur la question du visionnement du film et comment la 
convention occidentale prescrit aux spectateurs de se garder de dialoguer avec l'écran et avec les autres spectateurs, mais de regarder le film dans le silence. Ceci parce qu'à l'origine le film, contrairement à d'autres formes de divertissement populaire (songeons par exemple aux matchs de football et aux concerts de rock), a été conçu comme une expérience idiosyncratique et non communielle. Bouchard (2010:95) entrevoit la possibilité d'un autre regard sur le film, une autre façon d'engager le grand écran en écrivant : "In the popular forms of film projections, the brouhaha and various audience activities modify the reception of the film shown. At times, the interaction between the spectators and the film allows for appropriation. "

18 Au Congo Belge et partout en Afrique où le western figurait à l'affiche des salles de cinéma destinées aux jeunes, l'ambiance festive, à la limite du carnavalesque, avait de quoi choquer les Européens habitués à une audience plus passive. "Regarder un film », m'a confié une de mes sources, «c'était pire que le match de football. Ceux qui avaient vu le film avant revenaient pour commenter. On pouvait voir un film deux ou trois semaines d'affilée. On ne pouvait même pas suivre le dialogue. C'était surtout les actions que l'on suivait. C'était pire que le stade. C'était difficile d'imposer le silence $»^{13}$. L'historien de Kinshasa Molei Kolonga $(1979: 214)$ n'en dit pas moins, écrivant à l'époque où cette pratique animait encore les quartiers de Kinshasa : « Au moment de la projection, nous assistons à un spectacle d'un public en délire. Car à la première image du cow-boy, un ouragan d'applaudissements, une explosion de voix et des sifflements montent de la salle. Le dialogue sonore des films est englouti par les hurlements de spectateurs déchaînés, debout, qui, tantôt réclament davantage d'actions violentes, tantôt montés sur les sièges, poings fermés, se ruent sur des adversaires invisibles. »

19 Analphabétisme cinématographique railleront les missionnaires et certains évolués et journalistes congolais. Au contraire, tout indique des phénomènes d'appropriation et de détournement du film western. Dans la tradition du western, écrit Jane Tompkins $(1992: 51,56)$, action et silence servent à dérouler l'intrigue : quand les héros parlent ou se taisent, c'est de l'action. Le langage gestuel surtout, en véhiculant une image de maitrise de soi et le triomphe de la corporalité sur les émotions, finit par définir les contours d'un masculin sûr de soi, imperturbable et invulnérable. Faisant écho à Tompkins, Mitchell (1996: 165) observe de son côté : «Valuing action over words, marking silence as the most vivid of actions, the cowboy hero throws us back onto the male physique, shifting attention from ear to eye in the drama of masculinity. »

\section{Échos de la savane}

20 Le « bloc hybride », pour reprendre l'expression de Demetriou, dont les jeunes Kinois se servent dans les années 1950 pour construire leur masculinité, ne mobilise pas seulement les éléments empruntés au western hollywoodien. Il contient également les fragments d'un «masculin précolonial» mis à mal par les forces sociales et économiques de la colonisation. Par exemple, l'androgénisation des métiers domestiques au Congo Belge qui place les hommes dans des métiers auparavant réservés aux femmes - boys, jardiniers, cuisiniers et lavandiers - participe du projet de castration coloniale. Dans les lieux du travail, les magasins, les places publiques, le Noir se sent humilié par ces "tu», ces "sales macaques» et cette chicotte qui sans cesse l'essentialisent et disqualifient non seulement son humanité mais aussi sa masculinité14. 
21 Une certaine jeunesse dans les quartiers, que les rapports coloniaux qualifient de « délinquante » et de "désœuvrée », refuse en bloc la camisole de force que l'évolué doit enfiler pour apparaître homme aux yeux du colonisateur. Ces jeunes remuent le passé et en exhument des nkisi et moserebende qui respectivement les rendent invincibles lors des combats et leur permettent de séduire même les filles réputées « capricieuses ». L'organisation des bandes de cowboys à Kinshasa reflète également des dynamiques de genre qui prévalent dans la région avant l'arrivée des premiers Européens. Le chef de bande, par exemple, ne renvoie pas seulement à la figure surmasculinisée du sheriff ou du desperado tant célébrée dans le western. On peut y reconnaître également les traits et les gestes des chefs coutumiers comme Ingya, Ganshu, Gambiele, Makabi, Mubi et Ngako que décrivent les récits des explorateurs européens.

La structure des bandes imite la hiérarchie féodale du Pool colonial, avec les grands seigneurs comme Ngaliema et Makoko, de part et d'autre du Pool, qui mobilisent un capital mystique, militaire (grâce aux armes à feu qui pénètrent dans cette région à la faveur de la traite des esclaves) et lignager pour accroître leur prestige. Dans leurs huttes, ces grands seigneurs amassent d'énormes quantités de nkisi composés de « dents de lion, qui garantissent des bêtes féroces; graines trouées, qui préservent des maladies. On retire à tout animal mort ses dents, ses griffes et sa peau, et la personne qui porte sur elle ces objets n'a rien à craindre [...] des animaux vivants de la même espèce » (Guiral 1889: 178). L'anthropologue Robert Hottot qui visite le pays téké en 1906 remarque l'absence de ces «puissants fétiches» dans les huttes des femmes. La plupart des ingrédients auxquels ces chefs ont recours pour acquérir les attributs des animaux, force, rapidité, invulnérabilité, etc. (Hottot \& Willett $1956: 30$ ) entrent dans la composition des nkisi que les jeunes Kinois utilisent dans leurs kamôs (voir infra) dans les années 1950.

\section{Le Bill, ce bon Samaritain}

Au début des années 1950 des bandes de jeunes qui se donnent le nom de «Bills» (en hommage à leur héros éponyme Buffalo Bill) ou de «Yankees $»^{15}$ écument les quartiers de Kinshasa et adoptent l'allure, la morgue et le bagout du cowboy. Ils s'appellent Billy, Buffalo Bill, Texas Bill, Pecos Bill, Arizona, John Wayne, Burlan (diminutif de Burt Lancaster), Zorro, Tarzan, Hercule, Godzilla, Libre, Viking, Poison, 6600 Volts, Assassin, Mivais John, Amoureux Magique, Verre Cassé sans réparation, etc. ${ }^{16}$. Parmi eux, quelques Billesses, dont le nombre ne dépasse guère la douzaine, tentent de se couler dans le costume du « dur-à-cuir ». Traitées comme membres à part entière des bandes, leur féminité ne s'évanouit pourtant pas totalement mais est mise en jeu dans des stratagèmes dont ces bandes ont le secret ${ }^{17}$. L'une d'elle, Marie Cowboy, se lie d'amitié avec Mivais John dans le nouveau quartier satellitaire de Ndjili et ensemble jouent à Bonny et Clyde dans leur fief. Avant de joindre la bande d'Andrada au quartier Ruwet, Roy (plus connue sous son vrai nom de Thérèse Muyaka), se produisait sur la scène festive de Kinshasa dans la troupe de danse traditionnelle d'Alexis Kimbangu. Ses tours de reins virevoltants lors du zebola ${ }^{18}$ et son charme magnétique foudroient Andrada qui la courtise et parvient à l'enrôler dans sa petite bande de Bills. Andrada et ses compères se reconnaissent par leur tenue complète de cowboys, y compris des faux pistolets, que portent Thérèse et Meta, l'autre Billesse de la bande. 
Il importe de définir ces bandes de Bills, les contours du masculin qu'elles adoptent face au complexe colonial, leur subversivité et les activités souvent «infrapolitiques ${ }^{19}$ qui émaillent leur confrontation avec les autorités, qu'elles soient parentales ou politiques. Les Bills, dont certains après leurs quatre cents coups, se sont avantageusement recyclés dans la politique, l'armée, le sport et la musique, célèbrent la figure du Bill comme une sorte de "social bandit » dont l'historien marxiste anglais Eric Hobsbawn a été le premier à théoriser le portrait dans son ouvrage intitulé Primitive Rebels, paru en 1959. Le bandit social tel que l'analyse Hobsbawn dans l'Europe du XIX siècle et l'historien américain Richard White (1981) dans l'Ouest américain aux lendemains de la Guerre civile cristallise les résistances populaires vis-à-vis d'un ordre social souvent jugé injuste, voire oppressif. Leurs " crimes sociaux », hold-up, contrebande, piraterie, vandalisme et trafics en tous genres, qui donnent lieu souvent à une «justice redistributive » à la Robin des Bois, leur valent l'adulation des masses qui n'hésitent pas à les protéger. Voici donc comment un ancien Bill, musicien de renom, définit cette figure contestée :

«Un Yankee c'est un éducateur et un justicier. Quelqu'un qui fait des troubles est un faux Yankee. Il y avait des vrais et des faux Bills. Bill azali mutu ya normal [Le Bill est quelqu'un de normal]. Alingi mosala na ye [il aime faire son travail]. Les filles nous suivaient parce qu'on allait à l'école et qu'on jouait à la guitare. Les faux Yankees venaient nous attaquer parce qu'ils n'arrivaient pas à avoir des filles. Les Yankees allaient à l'école, comme Vieux Kabaidi, un vrai Yankee qui est allé à l'école et à l'université. Les Bills on les retrouvait dans tous les secteurs. Il y a des universitaires, des non-universitaires, des veilleurs de nuit. [...] Les Bills étaient des patriotes, des gens qui aiment le développement de leur pays. Les faux sont arrivés pour salir notre idéologie. C'est une idéologie de travailleurs, de gens qui aiment leur pays et leur prochain. Tu viens et tu trouves ton ami en train de manger? Il prend son manioc et le partage avec toi. Tu ne peux pas laisser ton ami crever de faim. Nous étions pour la solidarité. À l'école on partageait notre nourriture avec les plus petits $»^{20}$.

Paul Kabaidi, ancien gouverneur de la ville de Kinshasa, fait écho à cette longue évocation de Roitelet dans une surenchère hagiographique dictée par la mauvaise réputation dont ont hérité les Bills :

«Être Bill c'est quand vous demandez à quelqu'un d'être un homme. C'est être un homme. Moi je suis un Bill et fier de l'être. C'est l'homme droit, qui défend les justes causes, qui ne recule pas devant un danger, qui sait défendre les faibles, qui s'investit. On ne peut pas prendre ce mot dans le sens péjoratif qu'on lui a donné » 21.

26 Néron, né vers 1935 et dont le surnom ne provient pas d'un western mais d'un péplum, offre, dans un entretien qu'il m'a accordé deux ans avant sa mort, une vision du Bill dans laquelle se télescopent des notions bibliques et des relents eschatologiques post-11 septembre 2001. Selon lui, les vrais Bills étaient des « bons Samaritains » tandis que les faux étaient des "terroristes" qui semaient la zizanie et la peur dans les quartiers en commettant vols, viols et parfois même meurtres ${ }^{22}$.

27 À l'époque, chaque quartier de la capitale possède sa bande de Bills qui recrute activement et tente de veiller à l'intégrité de plusieurs frontières; celles de leur territoire d'abord ${ }^{23}$, menacées sans cesse par les bandes rivales qui tentent d'étendre les limites de leur influence et de séduire ou, à défaut, ravir les jeunes filles du quartier. De ce fait, les Bills se dressent comme un rempart contre ces intrusions et protègent jalousement l'hymen des filles de leur quartier. Les Bills veillent également aux 
frontières de genre dans leur propre fief en punissant les filles qui, dans leur argot, "vagabondent» ("kolúka») et, donc, se dévergondent. En indoubill, l'argot des Bills, kolúka subvertit l'ordre de genre et déplace la ligne de faille entre le masculin et le féminin. Le masculin pour les Bills est synonyme de mobilité et de visibilité, d'ubiquité et d'hubris. Le féminin, quant à lui, doit se cantonner à un script qui n'autorise ni mouvement ni agency (sens de l'action et du pouvoir). Kolúka dissimule mal des sousentendus sexuels expliquant les nombreuses volte-face du Bill qui oscille entre protecteur et prédateur, entre bon Samaritain et terroriste. Une fille du quartier qui traîne une réputation d'être « hautaine ", celle qui ne daigne pas répondre aux missives explicites envoyées par un grand Bill par l'entremise de son fidèle et loyal "secrétaire », ou celle que l'on accuse de flirter avec un Bill du quartier rival encourt l'ire des Bills qui trouve souvent son exutoire dans les tournantes endémiques (" éboulements ») leur attirant les foudres des « adultes » et des autorités ${ }^{24}$.

Cette versatilité du Bill, caractéristique qu'il partage avec l'archétype du «bandit social », suscite des attitudes ambivalentes de la part des autorités et des adultes. Leur rôle de " grand frère " auprès des enfants du quartier, qui par peur d'être réprimandés réfléchissent à deux fois avant de commettre des impairs (faire l'école buissonnière, par exemple), les fait entrer dans les bonnes grâces des Pères blancs. La surveillance qu'ils exercent sur les jeunes filles et l'encadrement des plus petits sur lesquels beaucoup de parents commencent à perdre l'emprise dans les années 1950 les rend incontournables. Les pratiques délinquantes dans lesquelles il faut ranger non seulement les tournantes (" éboulements») mais aussi la consommation de chanvre, l'idiome gouailleur et la tenue vestimentaire (le col de la chemise relevé indique leur rébellion), écornent l'image de « bon Samaritain » que les Bills entretiennent.

\section{Mama alobaki...}

Dans son étude sur la représentation de l'Ouest américain dans les films, l'historienne écossaise Jenni Calder (1974: 197) raconte que le père de John Wayne, sans doute inspiré par Shakespeare ${ }^{25}$, conseillait à son fils de ne pas chercher noise. "Mais si jamais tu te retrouves dans une bagarre » sermonnait-il, «fais tout pour gagner ». Cette remontrance, naturalisée dans le western comme le mantra du cowboy, fait surface chez les Yankees de Kinshasa et témoigne sans doute de l'intemporalité du masculin viril. À Kinshasa, dans le zeitgeist des années 1950, lorsqu'un enfant regagne le domicile la mine déconfite, après avoir essuyé une raclée, il se trouve toujours quelqu'un dans sa parcelle pour lui rappeler que "Mama alobaki, soki moninga abeti yo, yo pe obeti ye" ( "Mama a dit, si un camarade te tape, toi aussi tu dois le taper »). Il n'est pas lieu ici d'analyser ce qui saute aux yeux, l'asymétrie de genre entre le père de John Wayne, garant de sa masculinité, et la mama congolaise dont l'admonition peut paraître presque comme un palimpseste, un décalque du premier. Ce qui importe de dégager à travers cette transposition, si tant est qu'il y en ait une, est sa contribution au rituel du masculin qui s'établit fermement à Kinshasa dans les années 1950.

Tout jeune garçon dans les quartiers doit se forger un tempérament de caïd, tester les limites de sa virilité dans des épreuves de force quasi quotidiennes et se tenir prêt, sans jamais battre en retraite, à mobiliser le corps dans la défense du quartier, de sa famille et de sa masculinité. Pour s'assurer la victoire, la plupart des jeunes des quartiers, 
même ceux qui refusent de devenir membres d'une bande, adhèrent au traitement du corps auquel les Bills s'astreignent.

31 Au premier chef figure l'inévitable kamô (appelé aussi bilayi) qui confère aux Bills leur badge de masculinité. Pour moins de cinq francs (une bagatelle à l'époque) les Bills se rendent chez des grands Bills comme Degazin, Debarron, Eboma, Moruna (connu sous le nom de " roi du kamô ») et Verre Cassé pour obtenir des kamôs ${ }^{26}$. Le rituel consiste en plusieurs séquences. D'abord, à l'aide d'une lame de rasoir, le maître des kamôs opère deux petites incisions parallèles (nzoloko) sur le candidat, aux chevilles, torse, poignets et tempes. Ensuite, selon l'effet souhaité, le maître réduit en poudre, en les faisant cuire dans une petite poêle en fonte, dents et griffes de lion, poils de gorille, arêtes et peau d'un poisson visqueux nommé mina en lingala, mais aussi écorces d'arbres et plantes médicinales. Ainsi obtenue, la mixture (nkisi) est appliquée au nzoloko. Lorsqu'un client souhaite acquérir le pouvoir de donner des coups de tête "mortels", Degazin ajoute dans la poêle des morceaux de lames de rasoir, des clous et des aiguilles. Président de l'Union nationale des guérisseurs du Congo (UNAGCO) depuis la fin des années 1980, Degazin a appris le métier chez Eboma (qu'il appelle affectueusement « Vieux Ebo ») qui coupait les kamôs au quartier Mofewana (ou Far West). Degazin ouvre sa propre officine au quartier Saint-Jean (aujourd'hui Lingwala) où, me confie-t-il, il reçoit près de cinquante clients par jour. Pour aider ses clients à choisir leurs kamôs, il met à leur disposition un catalogue comprenant vingt-sept types de mixture ${ }^{27}$.

"Tout le monde a eu des kamôs", se souvient Hugor Bomina-Nsoni, dernier ambassadeur nommé par le président Mobutu à l'oNu, Bill durant sa jeunesse à Casamar ou Citas, qui fait aujourd'hui partie de la commune de Barumbu à Kinshasa.

«Pour donner des coups de tête, pour ceci, pour cela. Moi j'en ai eus. Tout le monde. Tout le monde y croyait. Moi j'ai vu des gens manger des tessons de bouteille. Tout le monde est passé par là $\rrbracket^{28}$.

33 Le pouvoir exercé par le kamô sur les Bills s'affirme à la fois comme physique et mystique. Il sanctuarise le corps masculin et le rend inviolable en créant autour de lui une frontière invisible. Même si les récits qui entourent le rituel du kamô peuvent paraître pour le moins apocryphes, ils dévoilent la tension qui taraude la construction du masculin à Kinshasa, tension entre destruction du corps (et donc destruction de soi) et préservation du corps. Une fois les kamôs coupés et le nkisi administré, le Bill entre en transe et se met à trembler de tout son corps. C'est le moment attendu par le maître, enhardi par la puissance de ses kamôs, pour casser des bouteilles sur la tête du client et lui en faire avaler les tessons. D'autres amènent carrément des lames de rasoir neuves qu'ils mastiquent et avalent en pleine transe, parfois devant un public médusé. Un certain Chege avale la cartouche d'un revolver Mauser en s'aidant de plusieurs gorgées d'huile de palme. Poison relate ainsi plusieurs séances qu'il a subies à Brazzaville à l'instigation de son masta (pote) dont le nom, Marteau, est à lui seul tout un programme :

« Dangwa les coupait aussi. Nous étions à deux parcelles de lui. Après avoir coupé on nous faisait avaler des choses. Moi j'ai avalé trois fois neuf aiguilles, 27 aiguilles. Je suis venu moi-même avec ces aiguilles. Je les avais achetées. C'est mon ami Marteau qui m'entraînait dans ces histoires-là. Il a fermé cela dans des fils et m'a dit de les avaler avec de l'eau avant de me couper les kamôs. Aujourd'hui je ne peux pas prendre cela. Je me demande où ces aiguilles sont parties dans mon corps. C'était à Brazza. J'ai fait cela en trois séances $»^{29}$. 

heureusement, ont survécu. Dans l'un d'eux, pose un Apollon du quartier Citas en compagnie de deux nzele ${ }^{33}$. La dichotomie de genre et la duplicité du jeune homme donnent matière à épiloguer. Lui, le torse dénudé et gonflé, exécute une pose de pugiliste, l'air triomphant, le regard vif; elles, parées dans des accoutrements qui voilent leurs formes et dévoilent l'image de la femme congolaise docile et soumise, le contemplent, comme envoutées par la mystique d'un dieu grec. Tandis qu'il tient la main de l'une, notre Apollon n'a pourtant d'yeux que pour l'autre. La composition de Depara, lui-même Bill durant sa jeunesse, recycle plusieurs poncifs qui nous sont maintenant familiers : au Bill est dévolu le beau rôle, un rôle de protecteur primé par le regard conquis de ses deux compagnes qui, loin d'admirer le corps, exprime plutôt une gratitude confinant à la dévotion. Derrière cette image candide se cache pourtant la sordide réalité d'un rapport de genre qui n'atteint son apothéose que dans une autre sorte de aufheben, représentée ici simultanément par la protection et la prédation des filles.

38 Le chanvre, auquel les Bills ont consacré le plus clair de leur vocabulaire, constitue la seconde activité. Il est dénommé communément zoumbel, nua ou diato ${ }^{34}$. Un des 
premiers écrits coloniaux à alerter les autorités sur la consommation du chanvre par la jeunesse kinoise est un rapport détaillé rédigé par Louis Bissot (2009 [1958]), un Belge commis à la section "problèmes de chômage " du Service de la population noire à Kinshasa. Bissot infiltre le milieu des Bills et mène l'enquête. Lorsque son rapport paraît en 1958, les autorités coloniales le mettent immédiatement en quarantaine, puis décident de confisquer et passer au pilon la plupart des exemplaires distribués dans les services du gouvernement ${ }^{35}$. Que reproche-t-on au rapport Bissot jusqu'à en interdire la diffusion? Au-delà de la description sordide des bas-fonds de Kinshasa et du désarroi d'une jeunesse abandonnée en jachère, c'est surtout l'approche délibérément empathique qui choque. Habituées à traiter "les problèmes de la jeunesse indigène " dans une optique stigmatisante et criminalisante, les autorités coloniales se cabrent devant l'idée qui attribue en grande partie la « délinquance juvénile » des jeunes Kinois à l'échec du modèle de socialisation et d'économie prôné par les Belges au Congo.

Bissot focalise son attention sur une bande de Bills au nom iconique de "Cowboys du Far West ». Cette bande s'était formée à Kintambo en 1950 lorsque Vieux Paurret connu premièrement sous le nom de Monganga (guérisseur) grâce à son activité de coupeur de kamôs - décida d'encadrer les jeunes déscolarisés du quartier. En 1952, lorsque la bande entre dans le collimateur de la police, Paurret règne en maître absolu à Kintambo grâce à ses kamôs qui font trembler, l'ordre de genre qu'il y établit tenant en respect les filles du quartier sous un véritable couvre-feu, et grâce à la protection qu'il offre aux jeunes du quartier. Son ubiquité, grâce à son vélo "Silver " ${ }^{36}$ qu'il conduit comme un étalon, fait rentrer les têtes brûlées dans le rang. Son autorité est telle qu'il parvient à subdiviser la commune de Kintambo en trois quartiers qu'il dénomme Kintambo-Lumière, Kintambo-Chicago et Kintambo-Assassin, noms qu'ils ont conservés jusqu'à présent. Paurret systématise le rituel du chanvre comme un critère pour faire partie de la bande des Cowboys. Fumer le chanvre ensemble, comme participer aux « éboulements ${ }^{37}$ qu'il organise, non seulement fait passer ces jeunes $d u$ côté des hommes mais crée un lien identitaire et leur permet de partager une expérience commune du masculin, de communier sous deux espèces: le sexe et la drogue.

40 Ces deux espèces, auxquels il faut ajouter le kamô, conduisent ces jeunes à «narcotiser » et à sublimer le corps, mitigeant ainsi l'impact de la violence coloniale qui souvent les touche de plein fouet. Mais comme Alcinda Honwana et Filip de Boeck (2005: 11) font bien de le remarquer, les jeunes performent le masculin dans une posture pendulaire, alternant entre makers (faiseurs) et breakers (casseurs) de normes sociales et culturelles qui régissent leur société. Ils actent comme ferments d'indiscipline, façonnent la culture autour d'eux mais se retrouvent souvent emportés par les lames de fond qui secouent leur société. Creuset où grâce à une véritable alchimie, les jeunes forgent leur masculinité, le corps n'en reste pas moins un site de subversion et de résistance, une sorte de redoute destinée à préserver l'homme de la tentation d'être autre chose qu'un homme.

\section{Yankee versus Yuma}

41 En se forgeant cette identité d'homme, de dur-à-cuir, le Bill le fait avant tout dans l'altérité. La figure qui le hante et dans l'envers de laquelle il s'évertue à se tailler un costume d'homme, en se faisant l'épigone du cowboy, n'est pas à proprement parler 
féminine même si elle possède certains traits que les Bills attribuent au "deuxième sexe ». L'antipode du Yankee est symbolisé par la figure pathétique du Yuma ${ }^{38}$. Être Yankee, c'est avant tout ne pas être Yuma, un terme dépréciatif qui évoque tous les attributs anti-mâles, le manque de courage avant tout, la naïveté ensuite et, de manière générale, l'absence de classe. Obnubilés par cette figure ignominieuse, Durango et quelques-uns de ses camarades décident de joindre une bande de Bills à KintamboAssassin :

« Pour nous, nous pensions que si on ne t'appelle pas Bill alors tu es Yuma. Il y avait une grande différence entre Yuma et Bill. Yuma c'était quelqu'un qui était naïf et que l'on dupait tout le temps. Être Bill, c'est être éveillé ${ }^{39}$.

La hantise d'être (mé)pris pour un Yuma pousse les jeunes des quartiers, même les plus studieux, à adopter la démarche du Bill, son franc-parler et ses airs de John Wayne.

«À cette époque-là si ton modega [col de la chemise] n'est pas en l'air, alors tu es pris pour un Yuma. À cette époque, une fille ne peut pas t'aimer. Elle te prend pour un Yuma. Il fallait mettre la boucle de côté. Changer la démarche et pencher une épaule en marchant. Là on ne peut pas venir te déranger parce qu'on sait que tu es un Bill et que tu vois clair $»^{40}$.

43 Certains jeunes se mettent sous la tutelle d'un grand Bill et, en échange de quelques pièces et d'une loyauté à toute épreuve, apprennent les rudiments du masculin, fument leur premier joint, se font couper des kamôs, et livrent leur premier combat pour un oui ou pour un nom, pour une fille ou pour rien. La peur d'être sans cesse tancé par un grand Bill, d'être poursuivi par la réputation de Yuma ou, pis, embousou (retardé), ngotobobo ou ngorobo (demeuré) pousse même les fils de bonne famille à orbiter autour des grands Bills ${ }^{41}$.

Yankees et Yumas se toisent dans les rues des quartiers. Le dénouement de ces confrontations est toujours dénué de suspens. Même quand ils n'en viennent pas aux mains, le Yankee a souvent le dessus avec son sens de la répartie et de la performance, ses injures qui blessent plus qu'un coup de poing. Penser que le football, dont les vertus communielles ne sont plus à prouver, amène Yankees et Yumas à se côtoyer dans les mêmes équipes serait une erreur manifeste. C'est tout le contraire qui se produit sur les terrains de fortune où les jeunes en décousent. D'abord, les Yankees refusent de se mélanger avec les Yumas, mais forment leurs propres équipes dont les noms, «Bombardier» ou "Terreur ", deux parmi d'autres, voilent à peine leurs desseins belliqueux. Ensuite, les règlements de compte ternissent la plupart des matchs. Pendant que la plupart des enfants du quartier se morfondent d'ennui à la messe dominicale, les Bills se donnent rendez-vous sur leur terrain de prédilection pour jouer au football. On joue pendant plusieurs heures. Un peu avant midi, l'arbitre déserte le terrain. C'est l'heure du bikumu, c'est-à-dire un jeu sans règles où tous les coups sont permis et où le match finit par dégénérer en bataille rangée. Bikumu est ce que les Yumas redoutent le plus lorsqu'ils affrontent les Yankees au football. Lors d'un match opposant l'équipe du quartier "Hirondelles» contre celle de l'Armée du Salut, dénommée "Roitelets ", l'arbitre siffle contre un joueur des Hirondelles pour une faute flagrante dans la surface de réparation. Un joueur des Roitelets pose le ballon sur le point de penalty et s'apprête à tirer. Le gardien des buts des Hirondelles, Romain Mpia, s'écarte des buts et s'adosse crânement contre l'un des poteaux. Sachant bien que marquer le but ne peut que donner un prétexte aux Yankees pour se livrer au bikumu, le tireur des Roitelets tape sagement à l'extérieur du cadre ${ }^{42}$. 

pour intimider et débouter ces derniers du chemin qui mène au Graal de la masculinité, il existe une fascination mutuelle. Les Yankees prennent la mesure des prodromes de fin d'empire lorsque l'édifice colonial se fissure, ébranlé par les émeutes populaires de janvier 1959 auxquelles ils prêtent d'ailleurs leur esprit insurrectionnel (Gondola 1999 : 173). Tous comprennent à ce moment-là que l'élite du Congo de demain ne sortira pas de la rue mais des écoles missionnaires. Déscolarisés pour la plupart, les grands Bills, comme nous l'avons vu, font la ronde des quartiers et sévissent contre les jeunes qui jouent à l'école buissonnière. Les Yumas, quant à eux, dont l'éducation est prise en charge par les missionnaires blancs, ne cachent pas leur admiration pour les grands Bills de leur quartier et, à peu d'exceptions près, finissent par embrasser leur ethos masculin.

in de compte, il importe peu d'être ou ne pas être Yankee, d'exhiber des kamôs, d'avoir des épaules larges, des pectoraux saillants et des biceps gonflés. Ce qui importe est de se faire précéder, partout où l'on met son pied, par la réputation d'un dur-à-cuir, de créer l'épouvante à la seule mention de son nom, d'arborer un air de supériorité, et de sceller un combat parcimonieusement, en faisant l'économie de sa force. Un tel ethos du masculin, qui convoque à la fois les ressorts de l'esprit et du corps et épouse la notion de "performative excellence » à l'aide de laquelle nous avons commencé cet essai, continue à servir de balise pour beaucoup de jeunes Kinois frappés par une crise qui est aussi une « crise » de masculinité.

Le «billisme » s'étiole au début des années 1960 puis s'éteint vers 1965 mais non sans passer le flambeau à d'autres mouvements de jeunes auxquels Tshikala Biaya (2000) a consacré une étude essentielle avant sa disparition prématurée en 2002. Au nombre des facteurs qui précipitent cette fin, il faut ranger l'action d'un missionnaire belge, Jozef de Laet (baptisé père Buffalo par les Bills), qui se lie d'amitié avec Billy, un grand Bill du quartier Dynamique à Ngiri-Ngiri. Père Buffalo pénètre le milieu Bill comme on entre dans les ordres, au grand dam de sa hiérarchie du CICM (Congregatio Immaculati Cordis Mariae). Il apprend l'indoubill (l'argot des Bills), songe même un temps à traduire la Bible en indoubill, communie avec les Bills dans la bière, le chanvre, la musique et le sexe, peut-être... disent les «mauvaises langues ». Au «Château Joc » qui leur sert de quartier général, à l'avenue Movenda, au quartier Dynamique, Père Buffalo initie les Bills à la maçonnerie, la cordonnerie et la mécanique. Les Bills y déjeunent pour la somme modique de 10 francs. Le sermon du Père Buffalo, chaque mardi soir, attire une foule plus large que d'ordinaire, y compris des Yumas qui viennent écouter ce Père blanc prêcher Christ en indoubill. À toute heure de la journée, les Bills y soulèvent des poids, y jouent au ping-pong et aux billes, pour les plus jeunes. On s'agglutine autour de Vieux Néron qui fait bouger les têtes et taper les pieds avec ses airs de guitare. On y fume aussi son joint sans la moindre inquiétude. Le Château possède sa propre boulangerie et les Bills recyclent leur cheval de fer (vélo), jadis chevauché à la cowboy, pour colporter et fournir du pain chaud à la ville. Père Buffalo canalise l'ardeur de certains Bills dans un magazine, Esprit de la jeunesse ${ }^{43}$, où des écrivains en herbe (dont l'un signe J. Victor Hugo dit Écrivain) s'essayent à brosser le nouveau portrait du Bill qui se dessine au Château.

« Vrai Bill il faut azala na bolingo [Le vrai Bill doit avoir l'amour] [...] azali na lolendo te

[il n'est pas arrogant], akoluka esengo ya ye moko te [il ne poursuit pas son propre 
bonheur] [...]. Le sacrifice du Christ sur la croix est la plus haute et la plus irrécusable preuve de l'amour du grand-bill (J.-C.) pour nous ${ }^{44}$.

\section{BIBLIOGRAPHIE}

AUSTIN, J. L.

1962 - How to Do Things with Words : The William James Lectures Delivered at Harvard University in 1955, Oxford, clarendon Press.

BHABHA, H.

1994 - The Location of Culture, London, Routledge.

BIAYA, T. K.

2000 - Les jeunes, la violence et la rue à Kinshasa : entendre, comprendre, décrire, Dakar, Codesria

(« Nouvelles Pistes »).

BILTEREYST, D. ET AL.

2012 - « Negotiating Cinema's Modernity : Strategies of Control and Audience Experiences of Cinema in Belgium, 1930s-1960s », in D. BILTEREYST et al. (eds.), Cinema, Audiences and Modernity : New Perspectives on European Cinema History, London, Routledge : 186-201. 
BISSOT, L.

2009 [1958] - Étude qualitative sur la délinquance juvénile à Léopoldville (ca. 1957), Rhode St. Genèse (Belgium), Paul Raymaekers Foundation (Abbreviated as the Bissot Report.)

BOUCHARD, V.

2010 - « Commentary and Orality in African Film Reception », in M. şAUL \& R. A. AUSTEN (eds.), Viewing African Cinema in the Twenty-First Century : Art Films and the Nollywood Video Revolution, Athens, Ohio University Press : 95-107.

BOURDIEU, P.

1998 - La domination masculine, Paris, Éditions du Seuil.

BURTON, A.

2005 - African Underclass : Urbanisation, Crime \& Colonial Order in Dar es Salaam, Athens, Ohio University Press.

CALDER, J.

1974 - There Must Be a Lone Ranger : The American West in Film and Reality, New York, Taplinger Publishing Company.

CARTER, R. A.

2000 - Buffalo Bill Cody: The Man Behind the Legend, New York, John Wiley \& Sons.

CONNELL, R. W.

1995 - Masculinities, Berkeley-Los Angeles, University of California Press.

CONVENTS, G.

2006 - Images \& démocratie : les Congolais face au cinéma et à l'audiovisuel. Une histoire politicoculturelle du Congo des Belges jusqu'à la République démocratique du Congo (1896-2006), Kessel-Lo (Belgium), Afrika Filmfestival.

CORNWALL, A. \& LINDISFARNE, N.

1994 - « Dislocating Masculinity : Gender, Power, and Anthropology », in A. CORNWALL \& N.

LINDISFARNE (eds.), Dislocating Masculinities : Comparative Ethnographies, London, Routledge : 11-47.

DEBORD, G.-E. \& WOLMAN, G. J.

1956 - « Mode d'emploi du détournement », Les Lèvres nues, 8 mai.

DEMETRIOU, D. Z.

2001 - «Connell's Concept of Hegemonic Masculinity : A Critique », Theory and Society, 30 : 337-61.

DIAMOND, A. J.

2009 - Mean Streets : Chicago Youths and the Everyday Struggle for Empowerment in the Multiracial City, 1908-1969, Berkeley, University of California Press.

GILMORE, D. D.

1990 - Manhood in the Making : Cultural Concepts of Masculinity, New Haven, Yale University Press. GONDOLA, C.-D.

1997 - Villes miroirs : Migrations et identités à Kinshasa et Brazzaville, 1930-1970, Paris, L'Harmattan. 1999 - « La contestation politique des jeunes à Kinshasa à travers l'exemple du mouvement "Kindoubill" (1950-1959) », Brood \& Rozen, Tijdschrift voor de Geschiedenis van Sociale Bewegingen, 2 : 171-183.

2009 - « Tropical Cowboys : Westerns, Violence, and Masculinity among the Young Bills of Kinshasa », Afrique \& Histoire, 7 : 75-98. 
GUIRAL, L.

1889 - Le Congo français : du Gabon à Brazzaville, Paris, Librairie Plon.

HERZFELD, M.

1985 - The Poetics of Manhood: Contest and Identity in a Cretan Mountain Village, Princeton, Princeton University Press.

HOBSBAWN, E.

1959 - Primitive Rebels : Studies in Archaic Forms of Social Movement in the 19th and 20th Centuries, New York, Norton.

HONWANA, A. \& DE BOECK, F. (EDS.)

2005 - Makers and Breakers : Children and Youth in Postcolonial Africa, Trenton, Africa World Press.

HOTTOT, R. \& WILLETT, F.

1956 - « Teke Fetishes », The Journal of the Royal Anthropological Institute of Great Britain and Ireland, 86 (1) : 25-36.

HOWSON, R.

2006 - Challenging Hegemonic Masculinity, London, Routledge.

KELLEY, R. D. G.

1994 - Race Rebels : Culture, Politics, and the Black Working Class, New York, The Free Press.

KOLONGA, M.

1979 - Kinshasa, ce village d'hier, Kinshasa, Sodimca.

MAISTRIAUX, R.

1957 - L'intelligence noire et son destin, Bruxelles, Les Éditions de Problèmes d'Afrique Centrale.

MITCHELL, L. C.

1996 - Westerns : Making the Man in Fiction and Film, Chicago, The University of Chicago Press.

MUTAMBA МАКОMBO KITATSHIMA, J.-M.

1998 - Du Congo Belge au Congo indépendant 1940-1960. Émergence des «Évolués » et genèse du nationalisme, Kinshasa, IFEP.

OMBREDANE, A.

1949 - « Principes pour une étude psychologique des Noirs du Congo Belge », L'année

psychologique, 50 : 521-547.

RAMIREZ, F. \& ROLOT, C.

1985 - Histoire du cinéma colonial au Zaïre, au Rwanda et au Burundi, Tervuren, Musée Royal de l'Afrique Centrale.

RAY, R.

1988 - The Men from the Boys : Rites of Passage in Male America, Lincoln-London, University of Nebraska Press.

RAYMAEKERS, P.

1963 - « Pre-delinquency and Juvenile Delinquency in Leopoldville », Bulletin de l'Institut InterAfricain du Travail, X (3) : 329-357.

ROTUNDO, E. A.

1993 - American Manhood: Transformations in Masculinity from the Revolution to the Modern Era, New York, BasicBooks. 
RYCKMANS, F.

2010 - Mémoires noires : les Congolais racontent les Congo Belge, 1940-1960, Bruxelles, Éditions Racines.

RYDELL, R. W. \& KROES, R.

2005 - Buffalo Bill in Bologna: The Americanization of the World, 1869-1922, Chicago, University of Chicago Press.

SADOUL, N.

1989 - Entretiens avec Hergé, édition définitive, Paris, Éditions Casterman (« Bibliothèque de Moulinsart »).

SOHN, A.-M.

2009 - Sois un homme ! Construction de la masculinité au XIX ${ }^{e}$ siècle, Paris, Éditions du Seuil.

TCHEBWA, M.

1996 - Terre de la chanson : la musique zaïroise hier et aujourd'hui, Louvain-la-Neuve, Duculot.

TOMPKINS, J.

1992 - West of Everything : The Inner Life of Westerns, New York, Oxford University Press.

UCHENDU, E.

2008 - «Introduction : Are African Males Men ? Sketching African Masculinities », in E. UCHENDU

(ed.), Masculinities in Contemporary Africa, Dakar, Codesria : 1-17.

VALE DE ALMEIDA, M.

1996 - The Hegemonic Male : Masculinity in a Portuguese Town, Providence-Oxford, Berghahn Books.

WHITE, R.

1981 - « Outlaw Gangs of the Middle Border : American Social Bandits », The Western Historical

Quarterly, 12 (4) : 387-408.

WHITEHEAD, S. M.

2002 - Men and Masculinities : Key Themes and New Directions, London, Polity Press.

\section{NOTES}

1. L'utilisation de termes en lingala, et non en français, pour qualifier la femme congolaise, termes que l'on retrouve sous la plume des missionnaires, des journalistes belges et des premières élites congolaises, indique que celle-ci, contrairement à l'homme noir, pouvait à peine échapper à l'indigénisation où l'avait confinée la «mission civilisatrice ».

2. À partir de 1936 toutes les agglomérations, grandes et moyennes, du Congo Belge héritent de ce statut qui les différencie des villages où les indigènes vivent sous «l'emprise de la coutume » (GONDOLA $1997:$ 116).

3. Les évolués, ces «demi-savants", moquait Monseigneur Rolens en 1941 (MUTAMBA мАКомво кітАТSнIMA 1998 : 43), correspondent au portrait fanonien dressé dans Peau noire, masques blancs, c'est-à-dire une classe composée de clerks, assistants médicaux, infirmiers, petits détaillants, agents comptables, instituteurs, prêtres, etc. qui, dans les années 1950, s'échinent pour obtenir des brevets de civilisation (qui servent également de badges de masculinité puisqu'il n'existe pas d'évoluée) que les Belges distribuent avec parcimonie. Il y en a à peu près 50000 en 1957 (sur une population totale de 13 millions de Congolais). Carte du Mérite civique (créée en 1948) et Carte 
d'Immatriculation (1952) récompensent les évolués qui s'astreignent à vivre comme des Européens. «On venait chez vous voir si vous aviez des fauteuils, si vous aviez un lit, si vous saviez tenir des fourchettes, si vos enfants portaient une culotte et des chaussures. On examinait votre intérieur [...] tout cela me révulsait » se souvient Joseph Mabolia (cité dans RYCKMANS $2010: 30$ ). Le nombre d'immatriculés n'atteindra pas 300 jusqu'à la fin de la colonisation.

4. Tentant de se disculper et de justifier ses croquis des Noirs peu flatteurs et les scènes racistes de son ouvrage, Hergé (alias Georges Prosper Remi) confiera avant sa mort en 1983, un demi-siècle après la première publication de Tintin au Congo: "Je ne connaissais de ce pays que ce que les gens racontaient à l'époque : "Les nègres sont des grands enfants, heureusement que nous sommes là !”, etc. » (cité dans SADOUL 1989 : 74).

5. Les jeunes ne s'y sont pas trompés, eux qui ont dans leur argot épinglé ces missionnaires blancs du terme ambigu de «mwasi mandefu» («femme barbue » ou, mieux encore, « femme à barbe »).

6. Même les évolués subissent un traitement des plus paternalistes lors des séances cinématographiques. On lit dans une note distribuée aux évolués lors d'une projection organisée en 1946 : « Spectateur, ce billet s'adresse à toi. Le Service de l'Information du Congo Belge veut te rendre service. Il a organisé cette séance de cinéma pour toi, évolué congolais. Il veut en organiser d'autres. " Suivent cinq questions demandant leur avis sur les films projetés, le prix d'entrée, etc. « Réponds à ces cinq questions ", lui intime la note. "Envoie ta réponse au Service de l'Information du Congo Belge à Léopoldville. Ne nous envoie pas une longue lettre, mais des renseignements précis. Nous voulons te donner des loisirs agréables et te permettre de continuer ta formation. C'est à toi de mériter la confiance des autorités » (cité dans RAMIREZ \& ROLOT 1985 : 182).

7. Lettre du Chef de zone A Croonenborghs à l'Administrateur de Territoire J. Cordy, Archives Africaines, Gouvernement Général (AA GG 19175), Bruxelles.

8. Paul Raymaekers, entretien avec l'auteur, Rhode Saint-Gènese, Belgique, 11 mai 2011. Témoin privilégié de l'histoire de Kinshasa, Paul Raymaekers est l'auteur de plusieurs travaux sur la délinquance juvénile à Kinshasa où il a résidé dans les années 1950 et 1960.

9. Les distributeurs américains semblent très tôt s'être intéressés au marché belge dont, en 1938, le Film Daily Year Book, une sorte d'almanach du cinéma, vantait la nonréglementation et l'absence de censure (BILTEREYST ET AL. 2012 : 187).

10. La popularité du Wild West Show fut telle que des jeunes garçons prirent d'assaut le bois de Boulogne et y plantèrent des tipis pour jouer aux cowboys et aux Indiens. À Bruxelles, Bologne, Munich et ailleurs en Europe l'engouement pour le Wild West Show a continué à donner lieu à des spectacles jusqu'à une période récente (RYDELL \& KROES 2005 : 109, 117).

11. D'ailleurs certains films missionnaires comme Le Rêve d'un grand roi: Léopoldville capitale du Congo Belge (1951) ne s'y trompent pas en représentant la capitale du Congo comme une sorte de Far West où la civilisation «moderne " et «nordique " terrasse l'empire des ténèbres (RAMIREZ \& ROLOT 1985 : 207).

12. Malgré la décision de la Commission de contrôle de censurer le film, jugé trop violent pour le public indigène, et de ne l'autoriser qu'au seul public européen, le film finit par circuler « sous le manteau » dans les quartiers africains.

13. Paul Kabaidi, entretien avec l'auteur, Bruxelles, 4 avril 2006. 
14. Lumumba raconte une scène vécue par beaucoup de Congolais pendant la colonisation. Dans la cohue de la rue, il bouscule une femme européenne. Celle-ci, sans lui donner l'occasion de s'excuser, lui lâche un «sale macaque!». Damas Tshiautwa (cité dans RYCKMANS 2010: 84) témoigne que, pour appeler n'importe quel Noir, l'expression «Hé macaque! Viens un peu ici!» était employée par de nombreux Européens. De ce point de vue, le discours de Lumumba le 30 juin 1960, aussi bien que le manifeste de Conscience Africaine qui l'a précédé le 30 juin 1956 (deux textes-phares de la décolonisation du Congo Belge) peuvent être considérés comme des manifestes d'émancipation et de masculinité. Dans l'un Lumumba déclare : «Qui oubliera qu'à un noir on disait "Tu", non certes comme à un ami, mais parce que le "Vous" honorable était réservé aux seuls blancs?» Dans le Manifeste, les auteurs congolais objectent: "Nous n'aimons pas toujours être traités comme des enfants.»

15. L'usage du terme «Yankee » précède celui de «Bill » de quelques années. Il remonte en effet aux années de guerre lorsque onze avions Dakota atterrissent à Kinshasa en 1942 dans le cadre d'une opération militaire destinée à convoyer des troupes américaines vers le front via le Congo. Au moins 1500 soldats américains sont casernés à Kinshasa jusqu'en 1943, au moment où le commandement américain transfère cette base d'opération au Maroc. Tandis que la déconfiture des armées belges et françaises crée une sorte de ressac colonial au sein de la population kinoise, se frotter de si près aux troupes yankees convoque une certaine mystique parmi ces jeunes Kinois qui les auréolent de gloire et tourbillonnent autour de leur campement. En échange de quelques babioles (souvent des vêtements usés, des cigarettes Lucky Strike, des bottes militaires, etc.), ces jeunes fournissent aux Yankees des compagnes d'une nuit. À cette époque, on ne parle plus lingala qu'en l'agrémentant de "yankee ", " mister ", " please ", "you come », "darling » et autres américanismes.

16. Certains de ces noms s'acquièrent comme des trophées et donnent lieu à des rivalités entre Bills et entre quartiers. N'est pas John Wayne qui veut, ni Buffalo Bill, ni Burlan, ni Zorro. Adopter un sobriquet déjà porté par un autre est un défi dont le dénouement ne survient qu'après un duel à la régulière. Le vainqueur remporte le nom tant convoité tandis que le vaincu devient la risée des quartiers.

17. Lorsque, par exemple, la bande est à court d'argent, le chef de bande répartit ses Billesses dans les bars pour jouer de leur charme, séduire et attirer des clients à l'extérieur du bar afin de permettre à la bande, postée en guet-apens, de les détrousser.

18. Traditionnellement pratiquée par les Mongo du Haut-Congo, la danse zebola se distingue par des mouvements saccadés par lesquels les danseurs des deux sexes accoutrés de guirlandes de raphia autour des reins rivalisent d'adresse pour " casser " les reins tout en restant dans le rythme.

19. J'utilise ce terme à l'instar de Robin KeLLey (1994) qui analyse la résistance de la classe ouvrière noire aux États-Unis en dehors du cadre proprement politique.

20. Roitelet (Augustin Moniania), entretien avec l'auteur, Brazzaville (Centre culturel français), 7 août 2006.

21. Paul Kabaidi, entretien avec l'auteur, Bruxelles, 4 avril 2006.

22. Néron (Joseph Katshaya Kopombo) et Petit Moloch (Abraham Koko Yawadio), entretien avec l'auteur, Kinshasa (Ngiri-Ngiri-Mofewana), 9 juillet 2005. 
23. Celles-ci consistent souvent en une rivière, une artère goudronnée (qu'on appelle " prince » parce que la toute première avenue, aujourd'hui Avenue Kasa-Vubu, avait été baptisée " Prince Baudoin ») ou un cimetière.

24. Paurret et Windy, les deux caïds de la bande "Cowboys du Far West », échouent à la prison de Ndolo avant d'être finalement appréhendés et relégués en résidence surveillée à l'intérieur de la colonie en 1956. Voici comment un rapport colonial décrit leurs frasques: "La bande s'abattait sur un marché volant du soir, poussant des cris effrayants, bousculant tout, et volant tout ce qui pouvait être emporté, parfois même des fillettes, qui étaient alors livrées aux appétits lubriques de tous les membres mâles. Vieux Pollet [sic], de son côté, arrêtait des filles à Brazzaville, les passait en pirogue avec la complicité d'un vagabond fils de pêcheur, et les livraient aux "clients" adultes de Léopoldville-Ouest, moyennant finance » (вISSOT 2009 : 18).

25. Dans la troisième scène du premier acte de Hamlet, Lord Polonius prodigue le même conseil à Laertes: "Beware of entrance to a quarrel; but being in, Bear't that the opposed may beware of thee. "

26. Brazzaville, le miroir de Kinshasa, de l'autre côté du fleuve, est réputé pour ses grands « féticheurs » et attire donc les Bills. Fantômas, qui a 22 ans en 1950, s'y rend régulièrement pour recevoir des kamôs d'un certain Makoubounzou au tarif d'un franc CFA; Fantômas (Mayoyo Gracia), entretien avec l'auteur, Kinshasa (Kasa-Vubu), 10 juillet 2005.

27. Degazin (Jean Sumbuka Bigonda), entretien avec l'auteur, Kinshasa (Kauka), 29 février 2009.

28. Hugor Bomina-Nsoni, entretien avec l'auteur, Kinshasa, 2 août 2009.

29. Poison (Jean-Christian Matabul), entretien avec l'auteur, Kinshasa (Lemba), 23 juillet 2009.

30. Chege (Baudoin Makenge), entretien avec l'auteur, Kinshasa (Kintambo-Chicago), 10 juillet 2005. Dans une autre version, tout aussi performative et attribuée à Vieux Ebo, dans laquelle on ne peut que noter l'influence du récit biblique, le tronc ne prend pas feu mais sèche, Fantômas (Mayoyo Gracia), entretien avec l'auteur, Kinshasa (KasaVubu), 10 juillet 2005 ; voir également TCHEBWA (1996: 124).

31. Kintulu provient du terme kikongo ntulu (adopté en lingala comme tolo) qui veut dire torse ou poitrine.

32. Voir la photographie en couverture de ce numéro.

33. Nzele (fille) provient du français «mademoiselle» ou plutôt de sa corruption, «mam'zelle». Les Bills truffent leur argot d'un éventail de termes, une preuve manifeste de l'importance des filles dans leur culture. On l'appelle également nzazi, momie, munini, mofide, nzoukoul, gonzo, popi, terd, tétard, mord, pétale, ngulufwa, kimwana mwana, petit mbongo, etc.

34. Paul RAYMAEKERS $(1963: 299)$ identifie dans l'argot des Bills au moins 75 termes ayant trait aux activités liées au chanvre. Voici, selon Petit Moloch, une des façons d'ajouter un nouveau terme au répertoire de l'indoubill : «Parfois on est assis entre nous et puis quelqu'un dit que les gens nous comprennent quand on dit nua. Bon, appelons ça diato à partir de maintenant », Petit Moloch (Abraham Koko Yawadio), entretien avec l'auteur, Kinshasa (Ngiri-Ngiri-Mofewana), 16 juillet 2007. 
35. En 2009, la Fondation Paul Raymaekers a reproduit, à partir d'un exemplaire original, et mis en vente le Rapport Bissot.

36. Friand de la série télévisée, Le Dernier des fédérés, Paurret baptise son vélo "Silver » comme le cheval du héros principal de cette série.

37. C'est en se frottant à celui que beaucoup considèrent comme l'ancêtre des Bills, un certain William Booth (arrêté en 1954 par l'inspecteur Maurice Derungs et détenu à la prison de Luzumbu dans le bas-Congo), dont il se vante d'avoir été le bras droit, que Paurret devient un « éboulementaire » endurci.

38. Le terme "Yuma» vient du film de Delmer Daves (1957), 3:10 pour Yuma, dont l'intrigue se déroule à travers un bras de fer psychologique entre un desperado, le chef de bande Ben Wade (joué par Glenn Ford), et un petit propriétaire de ranch, Dan Evans (que Van Heflin joue à merveille). La naïveté et la couardise des hommes de Contention City qui, un à un se défilent et abandonnent Evans à la mission périlleuse de convoyer Wade pour embarquer dans le train en partance pour Yuma, retient l'attention des Bills. Yuma devient donc synonyme de " peureux », « naïf » et « idiot ».

39. Durango (François Matouéni), entretien avec l'auteur, Kinshasa, 7 avril 2009.

40. Sylvain Eboma (Kutino Sylvain Miranda), entretien avec l'auteur, Kinshasa (Barumbu), 3 juin 2009.

41. José Patrick Nimy Mayidika Ngimbi, par exemple, qui commence sa carrière comme avocat à la Cour d'appel de Kinshasa en 1965 et finit conseiller spécial du président Mobutu entre 1990 et 1992, après avoir dirigé le bureau du président (1977-1986), s'est fait couper des kamôs par Degazin; Maître Nimy Mayidika Ngimbi, entretien avec l'auteur, Kinshasa (Ngombe), 20 mars 2009.

42. Jacques Atenda Mongebe Omwango, entretien avec l'auteur, Kinshasa (Gombe), 27 mai 2009.

43. Le magazine, qui atteint à son apogée un tirage mensuel de près de 5000 exemplaires, contient un éditorial et plusieurs rubriques, notamment «À travers les paroisses et quartiers » et " Actualités ». L'une d'elle, «Se mpo ya banzele » (réservée aux filles), témoigne de la normalisation des relations de genre qui définissent désormais le billisme.

44. J. Victor Hugo dit Écrivain, «Tolingana» («Aimons-nous les uns les autres »), Esprit de la jeunesse 14, 1964, p. 1.

45. Le mouvement jociste s'organise à Kinshasa à partir de 1931 sous l'égide du père scheutiste Frédéric Gangler. Il se donne pour mission d'aider les élèves des écoles professionnelles à entamer leur carrière professionnelle en leur instillant les valeurs catholiques.

\section{RÉSUMÉS}

Dans les années 1950, les jeunes des quartiers de Kinshasa embrassent la silhouette du cowboy à travers les films western qui dominent le projet cinématographique des missionnaires 
catholiques. Ces jeunes s'appellent Bills (à l'instar de leur héros éponyme Buffalo Bill), s'organisent en bandes, et adoptent les sobriquets, la dégaine, et le parler franc et gouailleur des cowboys écumeurs du Far West. Cet article s'intéresse donc à la construction des masculins à l'aide de rituels performatifs (souvent émaillés de violence) parmi les «cowboys tropicaux» de Kinshasa. Je m'attache particulièrement à démêler la question complexe de la résistance à travers la performativité et la culture populaire, en soulignant la manière dont ces jeunes produisent et actent leur identité par le biais du discours et de la performance. La problématique principale de mon étude s'articule autour de l'idée que le masculin et la quête de la virilité opèrent, au sein du même groupe interstitiel de jeunes, en suivant une trajectoire pendulaire dans laquelle la conservation du corps alterne avec son annihilation. Ainsi, la tension entre préserver et abolir la jeunesse, un mouvement qui correspond à la dialectique hégélienne de aufheben (négation de la négation), demeure précisément le point de bascule dans la quête du masculin tel que je l'examine parmi les jeunes de Kinshasa, un tournant où se joue le destin du masculin et où la virilité peut être gagnée ou perdue.

Manhood and The Cult of the Cowboy in 1950s Kinshasa

In the early 1950s, young people in Kinshasa's townships became infatuated with the silhouette of the cowboy which movies were the mainstay of the Catholic missionaries' cinematic venture. They dubbed themselves Bills (a nod to their eponymous hero Buffalo Bill), formed gangs, and adopted the moniker, the swagger, and the idioms of touch speech and ribald repartees of the Far West peripatetic cowboys. Hence, this paper addresses the construction of masculinities through performative rituals (oftentimes involving violence) among Kinshasa's "tropical cowboys". I am particularly interested in teasing out the complex issue of resistance through performativity and expressive culture, paying attention to the manners in which youths produce and enact their identity through discourse and performance. The central argument of the paper revolves around the idea that masculinity and the quest for manhood often operate, within the same interstitial group of youth, according to a pendulum process in which the construction of the body alternates with its annihilation. Indeed, the tension between preserving and abolishing youth, following Hegel's notion of aufheben (sublation), was precisely the tipping point in these young people's quest for masculinity, the make or break moment where manhood could be gained or lost.

INDEX

Keywords : Kinshasa, Belgian Congo, Buffalo Bill, Gangs, Cinema, Colonization, Cowboy, Genre, Young People, Masculinities, Missionaries, Violence, Western

Mots-clés : Kinshasa, Congo Belge, Buffalo Bill, bandes, cinéma, colonisation, genre, jeunes, masculinités, missionnaires, violence

\section{AUTEUR}

\section{CHARLES-DIDIER GONDOLA}

Indiana University, Indianapolis. 